\title{
Determinacy and the Structure of $L(\mathbf{R})$
}

\author{
ALEXANDER S. KECHRIS ${ }^{1}$
}

Let $\omega=\{0,1,2, \ldots\}$ be the set of natural numbers and $\mathbf{R}=\omega^{\omega}$ the set of all infinite sequences from $\omega$, or for simplicity reals. To each set $A \subseteq \mathbf{R}$ we associate a two-person infinite game, in which players I and II alternatively play natural numbers

$$
\begin{array}{lll}
\text { I } & x(0) & \\
\text { II } & x(1)^{x(2)} & x(3)
\end{array}
$$

$x(0), x(1), x(2), \ldots$ and if $x$ is the real they eventually produce, then I wins iff $x \in A$. The notion of a winning strategy for player I or II is defined in the usual way, and we call $A$ determined if either player I or player II has a winning stategy in the above game. For a collection $\Gamma$ of sets of reals let $\Gamma$-DET be the statement that all sets $A \in \Gamma$ are determined. Finally AD (The Axiom of Determinacy) is the statement that all sets of reals are determined.

As usual we denote by $L(\mathbf{R})$ the smallest inner model of Zermelo-Fraenkel (ZF) set theory which contains $\mathbf{R}$, i.e. the constructible from the reals universe.

If DC is the Axiom of Dependent Choice, which asserts that all binary relations with no finite descending chains are well founded, then it is easy to verify (using of course that DC holds in the universe $V$ of all sets) that $L(\mathbf{R}) \vDash D C$ as well. It has been proposed as a strong hypothesis of set theory that $L(\mathbf{R}) \vDash A D$, i.e. the Axiom of Determinacy holds in the realm of the sets constructible from $\mathbf{R}$. In this paper we give an overview of some recent results concerning the nature of this hypothesis and its implications for the structure theory of $L(\mathbf{R})$.

1. The equivalence of determinacy and partition properties in $\mathbf{L}(\mathbf{R})$. Except for $\S 2$ we will always work in $\mathrm{ZF}+\mathrm{DC}$ in the following. When stronger hypotheses are needed we will mention them explicitly.

1980 Mathrematics Subject Classification. Primary 03E47, 90D05, 90D13.

${ }^{1}$ Research partially supported by NSF Grant MCS81-17804.

(c) 1985 American Mathematical Society $0082-0717 / 85 \$ 1.00+\$ .25$. per page 
1.1. The study of the inner model $L(\mathbf{R})$ from AD splits into two seemingly different areas:

(a) descriptive set theory in an extended sense, i.e., the study of the structure of sets of reals in $L(\mathbf{R})$,

(b) "pure" set theory, i.e., the study of the structure of cardinals in $L(\mathbf{R})$. It turns out, however, that these two aspects of the theory of $L(\mathbf{R})$ are intimately related in a most subtle and surprising fashion. This intriguing interplay is our main subject in this paper.

A fundamental constant in the study of the continuum is the cardinal $\Theta$ defined as follows:

$$
\begin{aligned}
\Theta & =\sup \{\xi: \text { there is a surjection from } \mathbf{R} \text { onto the ordinal } \xi\} \\
& =\sup \{\xi: \xi \text { is the length of a pre-well-ordering on } \mathbf{R}\} .
\end{aligned}
$$

When we work in $L(\mathbf{R})$ (so that our underlying theory becomes $\mathrm{ZF}+\mathrm{DC}+V=$ $L(\mathbf{R})$ ), the structure of cardinals $>\Theta$ is well understood without any further hypotheses. This is because for such cardinals $L(\mathbf{R})$ behaves roughly as if it is an $L[A]$ for $A \subseteq \Theta$. This will be made precise and discussed in $\S 3$. So the analysis of cardinals in $L(\mathbf{R})$ from $\mathrm{AD}$ concentrates on those which are $\leqslant \Theta$.

1.2. The Axiom of Determinacy, even without assuming $V=L(\mathbf{R})$, leads to a rich structure theory of the cardinals smaller than $\Theta$. One of the important phenomena discovered is the existence of many cardinals below $\Theta$ with "large cardinal properties," for example measurability. Recall here Solovay's archetypical result that

$$
\mathrm{AD} \Rightarrow \boldsymbol{\aleph}_{1} \text { is measurable. }
$$

(For the basic results in descriptive set theory and the theory of determinacy we refer to Moschovakis' monograph [19], where references to the original papers can be found.)

Particularly important among these "large cardinal properties" are strong infinite exponent partition relations.

Definition. For an ordinal $\lambda$ and cardinals $\lambda \leqslant \kappa, \mu<\kappa$ the notation $\kappa \rightarrow(\kappa)_{\mu}^{\lambda}$ means that for every partition $F:[\kappa]^{\lambda} \rightarrow \mu$, of the set of increasing $\lambda$-sequences from $\kappa$ into $\mu$ pieces, there is a set $H \subseteq \kappa$ of cardinality $\kappa$, which is homogeneous for $F$, i.e., $F \uparrow[H]^{\lambda}$ is constant.

For infinite $\lambda$ the Axiom of Choice (AC) disproves the existence of such $\kappa$. Martin and Kleinberg originated in the late 1960s the study of infinite exponent partition relations in choiceless situations, in particular in the context of AD. Martin proved that

$$
\mathrm{AD} \Rightarrow \boldsymbol{\aleph}_{1} \rightarrow\left(\boldsymbol{\aleph}_{1}\right)_{\mu}^{\boldsymbol{\aleph}_{1}}, \quad \forall \mu<\boldsymbol{\aleph}_{1}
$$

a vast generalization of Solovay's theorem (see here [11 and 3]).

DEFINITION. A cardinal $\kappa$ has the strong partition property if $\kappa \rightarrow(\kappa)_{\mu}^{\kappa}, \forall \mu<\kappa$. 
Now in [7] it is shown that

$$
\mathrm{AD} \Rightarrow \forall \lambda<\Theta \exists \kappa(\kappa>\lambda \wedge \kappa \text { has the strong partition property }) .
$$

The main theorem in this section is the converse of this result in $L(\mathbf{R})$.

Theorem (Kechris and Woodin [8]). Assume $Z F+D C+V=L(\mathbf{R})$. Then the following are equivalent:

(i) $A D$,

(ii) there are arbitrarily large below $\Theta$ cardinals with the strong partition property.

This is the first purely set-theoretic formulation of the Axiom of Determinacy in $L(\mathbf{R})$. It emphasizes again the strong interconnections between this hypothesis and large cardinal properties of sets. It is worth pointing out here that by a recent result of Woodin (private communication) (ii) above does not imply alone (i.e. without $V=L(\mathbf{R})$ ) $\mathrm{AD}$, in facst it is consistent (modulo $\mathrm{ZF}+\mathrm{DC}$ ) with the existence of a nonprincipal ultrafilter on $\omega$.

1.3. Some of the key ingredients in the proof of this theorem are: (1) A uniform version of the Moschovakis Coding Lemma [19, p. 426] (needed for the direction (i) $\Rightarrow$ (ii) proved in [7]), which is, of course, ultimately based on the Recursion Theorem; (2) Steel's analysis of scales in $L(\mathbf{R})$ via the fine structure of this inner model (see [20]); (3) reflection properties of admissible sets and pointclasses; and finally (4) the Martin measure on the set of Turing degrees.

We briefly sketch now the main steps of the argument.

Part I. AD $\Rightarrow \forall \lambda<\Theta \exists \kappa(\kappa>\lambda \wedge \kappa$ has the strong partition property) (see [7]).

For each pointclass $\Lambda$ let

$$
o(\Lambda)=\sup \{\xi: \xi \text { is the length of a pre-well-ordering in } \Lambda\} .
$$

One shows that for certain $\Lambda$ 's, if $\kappa=o(\Lambda)$ then $\kappa$ has the strong partition property. These conditions on $\Lambda$ are reasonable enough so that every $A \subseteq \mathbf{R}$ belongs to at least one such $\Lambda$, so that these $\kappa$ 's are cofinal in $\Theta$. For instance it is sufficient to have $\Lambda=\Delta$, where $\Delta$ is the ambiguous part of a pointclass $\Lambda$ which contains all open sets and is closed under continuous preimages, countable intersections and unions, existential and universal quantification over $\mathbf{R}$ but not complements, and has the pre-well-ordering property. Given any $A \subseteq \mathbf{R}$, the pointclass $\Gamma=\mathbf{I N D}(A)$ of all inductive in $A$ sets has these properties and $A$ belongs to its ambiguous part $\Delta=\operatorname{HYP}(A)$ ( $=$ the hyperprojective in $A$ sets).

To show the strong partition property for $\kappa=o(\Delta)$, where $\Gamma$ satisfies the above conditions, one uses an appropriate uniform version of the Coding Lemma [19, p. 426] to code functions $f: \kappa \rightarrow \kappa$ by reals, and then associates to each partition $F$ : $[\kappa]^{\kappa} \rightarrow\{0,1\}$ (for simplicity into two pieces) a game $G(F)$ on $\omega$, in which apart from some side, but very crucial, conditions, players I and II collaborate to produce via this coding a function $f: \kappa \rightarrow \kappa$ and I wins iff $F(f)=0$. Say I has a winning strategy $\sigma$. Then using $\sigma$ one can construct a homogeneous set $H$ of cardinality $\kappa$ such that $F^{\prime \prime}[H]^{\kappa}=\{0\}$. The construction is by a boundedness 
argument in which the structural and closure properties of $\Gamma$ play a basic role. Symmetrically if II has a winning strategy, one can construct a homogeneous set $H$ of cardinality $\kappa$ with $F^{\prime \prime}[H]^{\kappa}=\{1\}$.

Part II. Assume $\mathrm{ZF}+\mathrm{DC}+V=L(\mathbf{R})$. Then if there are arbitrarily large below $\Theta$ cardinals with the partition property, AD holds.

Step 1 . Let us recall first a standard concept.

Definition. A set of reals $A$ is called Souslin if for some ordinal $\lambda$ there is a tree $T$ on $\omega \times \lambda$, with

$$
A=p[T]=\left\{\alpha \in \mathbf{R}: \exists f \in \lambda^{\omega} \forall n(\alpha \uparrow n, f \uparrow n) \in T\right\},
$$

where by a standard abuse of notation, for $s \in \omega^{n}, u \in \lambda^{n}$ we let $(s, u) \in T$ stand for $((s(0), u(0)), \ldots,(s(u-1), u(n-1))) \in T$.

One now has the following fact proved in [7]:

$$
\begin{aligned}
\forall \lambda<\Theta \exists \kappa(\kappa>\lambda & \wedge \kappa \text { has the strong partition property }) \\
& \Rightarrow \text { Every Souslin set of reals is determined. }
\end{aligned}
$$

For the proof let $A \subseteq \mathbf{R}$ be Souslin, say $A=p[T]$, where $T$ is a tree on $\omega \times \lambda$, where, of course, $\lambda$ can be assumed to be $<\Theta$. Then if

$$
T(\alpha)=\left\{u \in \lambda^{<\omega}:(\alpha \uparrow \text { length }(u), u) \in T\right\},
$$

we have

$\alpha \notin A \Leftrightarrow T(\alpha)$ is well founded

$\Leftrightarrow T(\alpha)$ with the Kleene-Brouwer ordering is well ordered.

For each $s \in \omega^{<\omega}$, let

$$
T(s)=\left\{u \in \lambda^{<\omega}: \text { length }(u) \leqslant \text { length }(s) \wedge(s \uparrow \text { length }(u), u) \in T\right\} .
$$

We think of $T(s)$ as well-ordered by the Kleene-Brouwer ordering.

To show that $A$ is determined choose first a cardinal $\kappa>\lambda$ satisfying the strong partition property. Then consider the auxiliary game in which player II also plays functions from various $T(s)$ into $\kappa$ :

$$
\begin{array}{lllll}
\text { I } & a_{0} & & a_{2} & \\
\text { II } & & a_{1}, f_{0} & & a_{3}, f_{2}
\end{array}
$$

and II wins iff each $f_{n}$ is an order-preserving map of $T\left(\left(a_{0}, a_{1}, \ldots, a_{2 n+1}\right)\right)$ into $\kappa$ and $f_{0} \subseteq f_{1} \subseteq f_{a} \subseteq \cdots$.

This is a closed game for player II, so it is determined. If II has a winning strategy he easily wins $\boldsymbol{A}$ by forgetting about the $f_{i}$ 's. If I has a winning strategy then use the partition property of $\kappa$ to make his moves independent of the $f_{i}$ 's and thus obtain a winning strategy for $\mathrm{I}$ in $A$.

Step 2. This is the main result of $[8]$.

THEOREM. Assume $Z F+D C+V=L(\mathbf{R})$. Then if every Souslin set is determined, every set is determined. 
This result has some relevance to the question of the plausibility of the hypothesis $L(\mathbf{R}) \vDash \mathrm{AD}$, since it shows that determinacy of arbitrary sets in $L(\mathbf{R})$ is reduced to that of "nice" sets, i.e., ones having the Souslin property in $L(\mathbf{R})$.

For a rough sketch of the proof, let $J_{\xi}(\mathbf{R})$ be the $\xi$ th stage of the Jensen hierarchy of $L(\mathbf{R})$. This is defined in the usual way relativizing the analogous one for $L$; for details see $[\mathbf{2 0}, \S 1]$. Abbreviate by $J_{\xi}(\mathbf{R})$-DET the statement that all sets of reals in $J(\mathbf{R})$ are determined. Then it is enough to show, assuming

$$
\mathrm{ZF}+\mathrm{DC}+V=L(\mathbf{R})+\text { Every Souslin set is determined, }
$$

that for every ordinal $\xi$,

$$
J_{\xi}(\mathbf{R}) \text {-DET } \Rightarrow J_{\xi+1}(\mathbf{R}) \text {-DET. }
$$

We recall first the following concept from Steel [20].

Definition. A $\Sigma_{1}$-gap is an interval $[\xi, \eta], \xi \leqslant \eta$, of ordinals such that $J_{\xi}(\mathbf{R})$ is a $\Sigma_{1}$-substructure of $J_{\eta}(\mathbf{R})$ for statements involving parameters in $\mathbf{R} \cup\{\mathbf{R}\}$ only, and $[\xi, \eta]$ is maximal with this property. We also view $\left[\sigma_{0}, \infty\right]$ as a $\Sigma_{1}$-gap, where $\sigma_{0}$ is the least ordinal $\sigma$ for which $J_{\sigma}(\mathbf{R})$ has this property relative to $L(\mathbf{R})$ itself.

The $\Sigma_{1}$-gaps partition the ordinals, so for an ordinal $\xi$ for which we want to prove $(*)$, let $[\eta, \zeta]$ be the $\Sigma_{1}$-gap containing $\xi$. We consider now cases.

Case $1 . \eta=\xi$, i.e. $\xi$ is the beginning of a $\Sigma_{1}$-gap.

Here we have two subcases.

Subcase 1.1. $J_{\xi}(\mathbf{R})$ is not admissible. Let then $\Gamma$ be the pointclass of all sets of reals which are $\Sigma_{1}$ over $L_{\xi}(\mathbf{R})$ with parameters in $\mathbf{R} \cup\{\mathbf{R}\}$ only. Then by [20, 2$]$, $\Gamma$ has the scale property, using $J_{\xi}(\mathbf{R})$-DET. Moreover, by nonadmissibility every $A \subseteq \mathbf{R}$ in $J_{\xi+1}(\mathbf{R})$ belongs to one of the classes $\forall^{\mathbf{R}} \Gamma, \exists^{\mathbf{R}} \forall^{\mathbf{R}} \Gamma, \forall^{\mathbf{R}} \exists^{\mathbf{R}} \forall^{\mathbf{R}} \Gamma$, etc. Since every set in $\Gamma$ being scaled is Souslin, we have $\Gamma$-DET, so the Second Periodicity Theorem [19, p. 311] applies to show that $\forall^{\mathbf{R}} \Gamma$ has the scale property, thus so does $\exists^{\mathbf{R}} \forall^{\mathbf{R}} \Gamma$ and by the same argument $\forall^{\mathbf{R}} \exists^{\mathbf{R}} \forall^{\mathbf{R}} \Gamma$ does as well, etc. So $A$ is Souslin, therefore determined. For obvious reasons we will refer to this method of showing that every $A \in J_{\xi+1}(\mathbf{R})$ is determined as the bootstrap argument.

TECHNICAL REMaRK. With the definition of $J_{\xi}(\mathbf{R})$ in [20] which starts with $J_{1}(\mathbf{R})=V_{\omega+1}$, this argument is not literally correct for $\xi=1$, since then $\Gamma=\Sigma_{1}^{1}$ and so $\Gamma$ does not have the scale property. But clearly the same bootstrap argument works in this case by starting instead with $\Gamma=\Sigma_{2}^{1}=\Sigma_{2}\left(J_{1}(\mathbf{R})\right)$, which does have the scale property (assuming just $\mathrm{ZF}+\mathrm{DC}$ ).

Subcase 1.2. $J_{\xi}(\mathbf{R})$ is admissible. Since $\xi$ is the beginning of a $\Sigma_{1}$-gap clearly $J_{\xi}(\mathbf{R})$ is $\Sigma_{1}$-projectible into $\mathbf{R}$, so we have to deal here with the following special case of $(*)$.

Key Lemma. Let $J_{\xi}(\mathbf{R})$ be admissible and $\Sigma_{1}$-projectible into $\mathbf{R}$. Then $J_{\xi}(\mathbf{R})$-DET $\Rightarrow J_{\xi+1}(\mathbf{R})$-DET.

The proof of this lemma is in ZF + DC only and does not use our hypothesis of determinacy of Souslin sets (in $L(\mathbf{R})$ ). The main idea is to start with an undetermined game $A \in J_{\xi+1}(\mathbf{R})$, write down explicitly the statement that it is not 
determined as a sentence in the first-order language of $J_{\xi}(\mathbf{R})$, and then "reflect" a suitable approximation of this statement down to $J_{\xi}(\mathbf{R})$, which can be used to cook up an undetermined game belonging to $J_{\xi}(\mathbf{R})$. For the implementation of this idea we use also a technique of Martin [16] for handling alternating strings of existential and universal quantifiers over $\mathbf{R}$ via the Martin measure on the Turing degrees. We refer to this method of showing games in $J_{\xi+1}(\mathbf{R})$ determined as the reflection method.

Case 2. $\eta \leqslant \xi<\zeta$. Then by the $\Sigma_{1}$-elementarity of $J_{\eta}(\mathbf{R})$ in $J_{\xi}(\mathbf{R})$, an undetermined game in $J_{\xi+1}(\mathbf{R})$ would give rise to an undetermined game in $J_{\eta}(\mathbf{R})$.

Case 3. $\eta<\xi=\zeta$, i.e., $\xi$ is at the end of a gap. Let $n$ be the least integer $\geqslant 1$ for which there is a new $\Sigma_{n}\left(J_{\xi}(\mathbf{R})\right)$ set of reals in $J_{\xi+1}(\mathbf{R})$. Then let us call following Steel [20, §3], [ $\eta, \zeta]$ a strong $\Sigma_{1}$-gap if every $\Sigma_{n}$-type realized in $J_{\zeta}(\mathbf{R})$ is also realized in some $J_{\zeta^{\prime}}(\mathbf{R}), \zeta^{\prime}<\zeta$. (By a $\Sigma_{n}$-type in any $J_{\rho}(\mathbf{R})$ we mean the set of all $\Sigma_{n}$ and $\Pi_{n}$ formulas satisfied by an element of $J_{\rho}(\mathbf{R})$ ). We now have two subcases.

Subcase 3.1. $[\eta, \zeta]$ is a strong $\Sigma_{1}$-gap. Then one can use a version of the reflection method (as in the proof of the Key Lemma) to show that if there is an undetermined game in $J_{\xi+1}(\mathbf{R})$, then there is already one in $J_{\eta}(\mathbf{R})$.

Subcase 3.2. [ $\eta, \xi]$ is not a strong gap. Then by Steel [20, §3], using $J_{\xi}(\mathbf{R})$-DET, we have that if $\Gamma=$ the pointclass of all $\Sigma_{n}\left(J_{\xi}(\mathbf{R})\right)$ sets of reals, $\Gamma$ has the scale property and every set of reals in $J_{\xi+1}(\mathbf{R})$ is in one of the following pointclasses: $\forall^{\mathbf{R}} \Gamma, \exists^{\mathbf{R}} \forall^{\mathbf{R}} \Gamma, \forall^{\mathbf{R}} \exists^{\mathbf{R}} \forall^{\mathbf{R}} \Gamma$, etc. Then by the bootstrap method of Subcase 1.1 we conclude that every $A \in J_{\xi+1}(\mathbf{R})$ is determined.

This finishes our sketch of the proof of the Theorem. A corollary of this proof is the following

Corollary. Assume $Z F+D C$. If there is a cardinal $\kappa$ with $\kappa \rightarrow(\kappa)_{2}^{\lambda}, \forall \lambda<$ $\Theta^{L(\mathbf{R})}$, then $L(\mathbf{R}) \vDash A D$.

This may be relevant to the problem of proving the consistency of the hypothesis that $\mathrm{AD}$ holds in $L(\mathbf{R})$ from appropriate large cardinal hypotheses.

2. The Axiom of Determinacy implies Dependent Choices in $L(\mathbf{R})$. The extensive theory of $L(\mathbf{R})$ from AD developed over the years provides a clear impression that " $\mathrm{ZF}+\mathrm{DC}+\mathrm{AD}+V=L(\mathbf{R})$ " is a "complete" theory for $L(\mathbf{R})$ in the similar vague sense in which " $\mathrm{ZF}+V=L$ " is thought of as a "complete" theory of $L$. There is one apparent difference however. Although $Z F+V=L$ implies $\mathrm{AC}$, one seems to need to add to $\mathrm{ZF}+\mathrm{AD}+V=L(\mathbf{R})$ the choice principle $\mathrm{DC}$ needed in several crucial places in the development of the theory of $L(\mathbf{R})$. However, as the next result shows this is not necessary.

Theorem [4]. $Z F+A D+V=L(\mathbf{R}) \Rightarrow D C$.

Thus one has the full analogy

$$
\frac{L}{Z F+V=L} \sim \frac{L(\mathbf{R})}{Z F+V=L(\mathbf{R})+A D} .
$$


As an immediate corollary we have the following solution to an early open problem in the theory of determinacy.

Corollary. $\operatorname{Con}(Z F+A D) \Rightarrow \operatorname{Con}(Z F+A D+D C)$.

It should be noted again that by a result of Woodin (see [4]) ZF + AD does not even imply the countable Axiom of Choice $\left(\mathrm{AC}^{\omega}\right)$. Thus $V=L(\mathbf{R})$ is necessary above.

A rough outline of the proof of the theorem is as follows. First notice that since $L=L(\mathbf{R})$, it is enough (assuming $\mathrm{ZF}+\mathrm{AD}$ ) to prove $\mathrm{DC}_{\mathbf{R}}$, i.e., $\mathrm{DC}$ for sets of reals. If now $\mathrm{DC}_{\mathbf{R}}$ fails, then it fails in $J_{\sigma_{0}}(\mathbf{R})$, where $\sigma_{0}$ is the least $\sigma$ for which $J_{\sigma}(\mathbf{R})$ is a $\Sigma_{1}$-substructure of $L(\mathbf{R})$ for statements involving parameters in $\mathbf{R} \cup\{\mathbf{R}\}$ only. To get a contradiction, it is enough to show that every relation $R \subseteq \mathbf{R} \times \mathbf{R}$ which belongs in $J_{\sigma_{0}}(\mathbf{R})$ can be uniformized. Since $J_{\sigma_{0}}(\mathbf{R})$ is $\Sigma_{1}$-projectible into $\mathbf{R}$, every $A \subseteq \mathbf{R}, A \in J_{\sigma_{0}}(\mathbf{R})$, is $\Sigma_{1}$-definable in $J_{\sigma_{0}}(\mathbf{R})$ using only parameters in $\mathbf{R} \cup\{\mathbf{R}\}$. So it is enough to show that the class of $\Sigma_{1}\left(J_{\sigma_{0}}(\mathbf{R})\right)$ sets of reals has the scale property, since scales imply easily uniformization. This follows immediately by Steel [20, §2], but unfortunately his proof uses DC. The way out of this vicious circle is to weaken the notion of scale, call it quasiscale, then show that Steel's proof goes through with this weaker notion without $D C$, and finally show that quasiscales are enough to prove uniformization.

3. The Continuum Problem in $L(\mathbf{R})$. The Continuum Problem asks for the place of the cardinality of the continuum $2^{\aleph_{0}}$ in the series of alephs (= initial ordinals, called cardinals in the sequel). Since assuming AD the set of reals cannot be well ordered, this formulation does not make sense in our context. On the other hand it is an early theorem of $\mathrm{AD}$, due to Morton Davis, that every set of reals is either countable or contains a perfect set. So a different interpretation of the Continuum Problem can be settled from AD.

Even in the absence of a well-ordering of $\mathbf{R}$, we can measure the "length" of the continuum by the cardinal

$$
\Theta=\sup \{\xi: \xi \text { is the length of a pre-well-ordering of } \mathbf{R}\} .
$$

Note that with AC, $\Theta=\left(2^{\aleph_{0}}\right)^{+}$. So we can reformulate the Continuum Problem as the question of the place of $\Theta$ in the series of cardinals. When understood this way the Continuum Problem leads naturally to the corresponding question about arbitrary pointclasses $\Lambda$. Let as before

$$
o(\Lambda)=\sup \{\xi: \xi \text { is the length of a pre-well-ordering in } \Lambda\} .
$$

Assuming we are in $L(\mathbf{R})$ and AD holds in that universe, it can be shown (see [19, p. 430]) that for most interesting $\Lambda, o(\Lambda)$ will be a cardinal. So we can ask for the place of $o(\Lambda)$ in the series of cardinals of $L(\mathbf{R})$. We will concentrate here on some particularly important examples of $\Lambda$ 's for which we recall the following more or less standard notation:

(a) $o(\operatorname{power}(\mathbf{R}))=\Theta$. 
(b) Let IND be the class of all inductive sets of reals (equivalently all sets of reals which are $\Sigma_{1}$ over the smallest admissible set $\mathbf{R}^{+}$containing $\mathbf{R}$ ), let $\mathbf{H Y P}=(\mathbf{I N D})^{\circ} \cap \mathbf{I N D}$ be the ambiguous part of IND (i.e. the hyperprojective sets) and let $o(\mathbf{H Y P})=\kappa^{\mathbf{R}}$. Clearly $\kappa^{\mathbf{R}}$ is the ordinal of the admissible set $\mathbf{R}^{+}$.

(c) Let $\mathbf{E N V}\left({ }^{3} E\right)$ be the class of all sets of reals which are (Kleene-) semirecursive in ${ }^{3} E$ and a real and let $\operatorname{SEC}\left({ }^{3} E\right)$ be the ambiguous part of $\operatorname{ENV}\left({ }^{3} E\right)$, i.e., the sets of reals which are recursive in ${ }^{3} E$ and a real. Put

$$
o\left(\operatorname{SEC}\left({ }^{3} E\right)\right)=\kappa^{K L} .
$$

(d) Finally let $o\left(\Delta_{n}^{m}\right)=\Delta_{n}^{m}$. Of particular interest are the projective ordinals $\delta_{n}^{1}$ and the ordinal $\delta_{1}^{2}$.

The order relationship between the above ordinals is

$$
\delta_{n}^{1}<\kappa^{K L}<\kappa^{\mathbf{R}}<\delta_{1}^{2}<\Theta
$$

Assuming AD they are all cardinals and $\delta_{n}^{1}<\delta_{n+1}^{1}$. We now proceed to discuss in more detail their properties.

3.1. The structure of $\Theta$ in $L(\mathbf{R})$. The following simple fact is proved in $\mathrm{ZF}+\mathrm{DC}+V=L(\mathbf{R})$ only.

(a) $\Theta$ is a regular cardinal.

Can $\Theta$ have any large cardinal properties? The result below, again proved in just $\mathrm{ZF}+\mathrm{DC}+V=L(\mathbf{R})$, imposes some severe limitations.

(b) THEOREM (Kechris AND WoOdIN [9]). The cardinal $\Theta$ cannot be weakly compact (for instance there is a $(\Theta, \Theta)$-tree with no $\Theta$-branch and thus $\Theta \rightarrow(\Theta)_{2}^{2}$ fails).

The proof of this Theorem uses among other things the following result, which extends a theorem of Vopenka (see Jech [2]).

Assume $\mathrm{ZF}+\mathrm{DC}+V=L(\mathbf{R})$. Then $\mathrm{HOD}=L[A]$, where $A \subseteq \Theta$. Moreover there is a notion of forcing $\mathbf{P}$ in HOD such that in HOD $\mathbf{P}$ has cardinality $\Theta$ (the $\Theta$ of the universe, not of HOD), and the $\Theta$-chain condition, and $L(\mathbf{R})$ is a symmetric generic extension of HOD via $\mathbf{P}$.

From this it follows that the cardinals and cofinalities of $L(\mathbf{R})$ above $\Theta$ are the same as those of $\mathrm{HOD}=L[A]$ (recall here our remarks in $\$ 1$ ). Also there are no measurable (or Ramsey cardinals) $\geqslant \Theta$ in $L(\mathbf{R})$.

On the other hand AD implies that $\Theta$ is very large.

(c) Theorem (Kechris And WoOdin [9]). Assume $Z F+A D+V=L(\mathbf{R})$. Then $\Theta$ is weakly $\Theta-$ Mahlo.

The proof of this Theorem uses recursion-theretic ideas. Let us show for instance that $\Theta$ is weakly Mahlo.

We first need the following simple choice Lemma. 
LEMMA (ZF $+\mathrm{DC}+V=L(\mathbf{R}))$. Let $f: \Theta \rightarrow V$ be such that $f(\xi) \neq \varnothing, \forall \xi<\Theta$. Then there is $A \subseteq \Theta, A$ of cardinality $\Theta$ and $g: A \rightarrow V$ such that $\forall \xi \in A$, $g(\xi) \in f(\xi)$.

Proof of The Lemma. It is enough to assume $f(\xi) \subseteq \mathbf{R}, \forall \xi<\Theta$, since there is a definable $F$ : ORD $\times \mathbf{R}^{\text {onto }} \rightarrow V$. But then we claim that there is an $A$ as above that $\bigcap_{\xi \in A} f(\xi) \neq \varnothing$, which finishes the proof. If not then for each $x \in \mathbf{R}$, $h(x)=\sup \{\xi<\Theta: x \in f(\xi)\}<\Theta$, so let $\lambda<\Theta$ be bigger than all the $h(x)$, $x \in \mathbf{R}$. Then $f(\lambda)=\varnothing$, a contradiction.

Now let, using this Lemma, $P: \Theta \rightarrow \operatorname{power}(\mathbf{R} \times \mathbf{R})$ be a function such that $P(\xi)$ is a pre-well-ordering of $\mathbf{R}$ of length $\xi$. Fix a function $g: \Theta \rightarrow \Theta$. We have to find a regular cardinal $\kappa<\Theta$ such that $g(\xi)<\kappa, \forall \xi<\kappa$. For that consider the following type 3 object on $\mathbf{R}$ :

For $X \subseteq \mathbf{R} \times \mathbf{R}, x, y \in \mathbf{R}$

$$
{ }^{3} F(X, x, y)= \begin{cases}0 & \text { if } X \text { is a pre-well-ordering of } \mathbf{R} \text { of length, say, } \xi \\
\text { and }(x, y) \in P(g(\xi)) & \begin{array}{l}
\text { otherwise. }
\end{array}\end{cases}
$$

Let $\Gamma=\operatorname{ENV}\left({ }^{3} E,{ }^{3} F\right)$ be the pointclass of all sets of reals which are semirecursive in ${ }^{3} E,{ }^{3} F$ and a real. Let $\Delta=\mathbf{S E C}\left({ }^{3} E,{ }^{3} F\right)$ be its ambiguous part and $o(\Delta)=\kappa$. Then by a result of Moschovakis (see [19, p. 430]) $\kappa$ is regular (in fact weakly inaccessible). If $\xi<\kappa$, fix a pre-well-ordering $X$ of length $\xi$ which is in $\Delta$, i.e., it is recursive in ${ }^{3} E,{ }^{3} F$ and a real. Then

$$
(x, y) \in P(g(\xi)) \Leftrightarrow^{3} F(X, x, y)=0,
$$

so $P(g(\xi))$ is also recursive in ${ }^{3} E,{ }^{3} F$ and a real, thus $g(\xi)<\kappa$.

Actually as in $\S 1$ it turns out that for $\Gamma$ as above, the corresponding $\kappa=o(\Delta)$ satisfies the strong partition property. By a result of Kleinberg [11] even if $\kappa \rightarrow(\kappa)_{2}^{\omega+\omega}, \kappa$ is a measurable cardinal, so every closed unbounded subset of $\Theta$ contains measurable cardinals. As a simple corollary we have the following purely set-theoretic description of $\Theta$ in $L(\mathbf{R})$.

(d) Assume $\mathrm{ZF}+\mathrm{AD}+V=L(\mathbf{R})$. Then $\Theta$ is the supremum of the measurable cardinals.

We conclude by mentioning that it appears that nothing is known about the consistency strength of $\mathrm{ZF}+\mathrm{DC}+V=L(\mathbf{R})+\Theta$ is large (e.g. weakly inaccessible, Mahlo, etc.).

3.2. Measures in $L(\mathbf{R})$. We will see that the study of the Continuum Problem in the context of $\mathrm{AD}$ is intimately related with the structure of measures on cardinals. Let us start with some basic facts.

(a) Theorem (Folklore). Assume $Z F+D C+A D$. Then every ultrafilter is countably complete. 
Otherwise there would be a nonprincipal ultrafilter on $\omega$, thus a nonmeasurable set of reals.

(b) Theorem (Kunen). Assume $Z F+D C+A D$. For each $\lambda<\Theta$, the set of ultrafilters on $\lambda$ is well-orderable.

Proof. By the Coding Lemma there is $F: \mathbf{R}^{\text {onto }} \rightarrow$ power $(\lambda)$. Let $\mathscr{U}$ be an ultrafilter on $\lambda$. Let $\mathscr{D}$ be the set of Turing degrees and define $f: \mathscr{D} \rightarrow \lambda$ by

$$
f(d)=\text { the least ordinal in } \bigcap\left\{F(x): x \leqslant_{T} d \wedge F(x) \in \mathscr{U}\right\} .
$$

Then if $\mu$ is the Martin measure on $\mathscr{D}$, i.e., the one generated by cones, $f_{*} \mu=$ def $\left\{X \subseteq \lambda: f^{-1}(X) \in \mu\right\}=\mathscr{U}$. Now for $f, g: \mathscr{D} \rightarrow \lambda$

$$
[f]_{\mu}=[g]_{\mu} \Rightarrow f_{*} \mu=g_{*} \mu
$$

so we can map $\lambda^{\mathscr{D}} / \mu$ onto the set of all ultrafilters on $\lambda$.

Let us introduce now the following

DeFinition. For each cardinal $\kappa, M(\kappa)=$ the set of all ultrafilters on $\kappa$, and $\beta(\kappa)=$ the cardinal of $M(\kappa)$.

This makes sense also in the context of AD by the preceding result.

Note that in the AC context $\beta(\kappa)=2^{2^{\kappa}}$. In the AD context $2^{\kappa}$ does not make sense as a cardinal, and $\beta(\kappa)$ is the closest analog to a power-type operation for cardinals that we can get. However the reader should be cautioned that the usual rules of power do not necessarily apply, e.g., we can have $\kappa$ with $\beta(\kappa)=\kappa$.

We will see that the Continuum Problem for pointclasses, in the way we have described it in the beginning, is intimately related with the behavior of the function $\beta(\kappa)$, whose computation can be understood as a version of the Generalized Continuum Problem in the context of AD.

The function $\beta(\kappa)$ allows us to define the concept of strong inaccessibility in a way that makes sense even with AD.

DeFINITION. We call a cardinal $\kappa$ strongly inaccessible if

(i) $\kappa$ is regular,

(ii) $\lambda<\kappa \Rightarrow \lambda^{+}<\kappa$,

(iii) $\lambda<\kappa \Rightarrow \beta(\lambda)<\kappa$.

Are there any such cardinals in $L(\mathbf{R})$ ? The following answers that positively.

(c) Theorem (Kechris [5]). Assume $Z F+A D+V=L(\mathbf{R})$. Then $\delta_{1}^{2}=o\left(\Delta_{1}^{2}\right)$ is strongly inaccessible.

On the other hand we have

(d) Theorem (Martin). Assume $Z F+A D+V=L(\mathbf{R})$. Then for $\delta_{1}^{2} \leqslant \kappa<\Theta$, $\beta(\kappa)=\Theta$, so $\Theta$ is not strongly inaccessible.

As a corollary we have the following set-theoretic characterization of $\delta_{1}^{2}$, in 
$\mathrm{ZF}+\mathrm{AD}+V=L(\mathbf{R}):$

$$
\delta_{1}^{2}=\text { the largest measurable stongly inaccessible cardinal. }
$$

3.3. The structure of $\kappa^{\mathbf{R}}$. We will characterize now the ordinal $\kappa^{\mathbf{R}}$, assuming just $\mathrm{ZF}+\mathrm{DC}+\mathrm{AD}\left(V=L(\mathbf{R})\right.$ is not needed here). It can be seen as in $\S 1$ that $\kappa^{\mathbf{R}}$ has the strong partition property, as in 3.1(c) that it is weakly Mahlo and as in $3.2(\mathrm{c})$ that it is strongly inaccessible. To obtain a precise set-theoretic description of $\kappa^{\mathbf{R}}$ we need the following notion of indescribability.

Definition. Let $B_{\lambda}=\left\{X: \exists \xi<\lambda\left(X \subseteq L_{\xi}\right)\right\}$ for each limit ordinal $\lambda$. Then $L_{\lambda} \subseteq B_{\lambda}$ and $B_{\lambda}$ is transitive. Call $\lambda$ weakly ${ }^{b} \Pi_{2}^{1}$-indescribable ( $b$ for bounded) if for each $X \subseteq L_{\lambda}$ and each $\Pi_{2}$ formula $\varphi$ with parameters in $B_{\lambda}$ we have

$$
\left\langle B_{\lambda}, \varepsilon, X\right\rangle \vDash \varphi \Rightarrow \exists \xi<\lambda, \quad\left\langle B_{\xi}, \varepsilon, X \cap L_{\xi}\right\rangle \vDash \varphi .
$$

Then by [6], $\kappa^{\mathbf{R}}$ is weakly ${ }^{b} \Pi_{2}^{1}$-indescribable. We now have

Theorem. Assume $Z F+D C+A D$. Then $\kappa^{\mathbf{R}}$ is the least cardinal $\kappa$ such that

(i) $\kappa$ is strongly inaccessible, and

(ii) $\kappa$ is weakly ${ }^{b} \Pi_{2}^{1}$-indescribable.

Conjecture. In this theorem (i) can be replaced by: $\kappa$ is weakly inaccessible.

3.4. The Kleene ordinal $\kappa^{K L}$. Again assuming $\mathrm{ZF}+\mathrm{DC}+\mathrm{AD}, \kappa^{K L}$ is a cardinal with the strong partition property and it is also strongly inaccessible. In fact, we have the following elegant characterization of $\kappa^{K L}$. It follows by combining results of Kechris [5], Martin, and Steel [21].

Theorem (Kechris, Martin and Steel). Assume $Z F+D C+A D$. Then $\kappa^{K L}$ is the least strongly inaccessible cardinal.

The following is an old conjecture in this area.

Conjecture (Moschovakis). $\kappa^{K L}$ is the least weakly inaccessible cardinal.

3.5. On the $\delta_{n}^{1}$ 's. From Moschovakis [19] it follows that $\delta_{n}^{1}$ is a regular cardinal. In fact, it can be shown that each $\boldsymbol{\delta}_{n}^{1}$ is measurable (Kunen [13], Martin [17]) and, in fact, each $\delta_{n}^{1}$ with $n$ odd satisfies partition properties with fairly large exponents (Kunen [14], Kechris and Woodin [10]), but it is not known whether any $\delta_{n}^{1}$ for $n>1$ odd has the strong partition property (the $\delta_{n}^{1}$ with $n$ even cannot, Kunen [12]). This is all assuming, of course, that $\mathrm{ZF}+\mathrm{DC}+\mathrm{AD}$. Here are two further general rules, under the same hypothesis:

(a) Assume $\mathrm{ZF}+\mathrm{DC}+\mathrm{AD}$. Then $\delta_{2 n+1}^{1}=\left(\lambda_{2 n+1}\right)^{+}$, where $\lambda_{2 n+1}$ is cardinal of cofinality $\omega$.

(b) (Kunen [15] and Martin [12]) Assume ZF + DC + AD. Then $\delta_{2 n+2}^{1}=$ $\left(\delta_{2 n+1}^{1}\right)^{+}$. 
So it is enough todetermine the $\boldsymbol{\delta}_{2 n+1}^{1}$ 's. The following are known:

$$
\left.\begin{array}{l}
\boldsymbol{\delta}_{1}^{1}=\boldsymbol{\aleph}_{1} \quad \text { (classical) } \\
\boldsymbol{\delta}_{2}^{1}=\boldsymbol{\aleph}_{2} \\
\boldsymbol{\delta}_{3}^{1}=\boldsymbol{\aleph}_{\omega^{+1}} \\
\boldsymbol{\delta}_{4}^{1}=\boldsymbol{\aleph}_{\omega^{+2}}
\end{array}\right\} \quad \text { Martin [18], in ZF+DC+AD. }
$$

The $\delta_{n}^{1}$ for $n \geqslant 5$ are not known in terms of the alephs. On the other hand, the $\delta_{n}^{1}$ 's are almost precisely computed in terms of the $\beta$ function, in view of the following result which generalizes an earlier theorem of Kunen [14] (for $n=0,1$ ). Its proof combines results of Becker [1], Kechris [5], Kunen [14], Martin and Woodin.

Theorem (Becker, Kechris, Kunen, Martin and Woodin). Assume $Z F+$ $D C+A D$. Then for each $n \geqslant 1$,

$$
\lambda_{2 n+3} \leqslant \beta\left(\delta_{2 n+1}^{1}\right) \leqslant \delta_{2 n+3}^{1} .
$$

Conjecture. For all $n, \beta\left(\delta_{2 n+1}^{1}\right)=\lambda_{2 n+3}$. (This is true for $n=0,1$ by Kunen.)

From the preceding results it is now clear that the final resolution of these aspects of the Continuum Problem in $L(\mathbf{R})$ and other models of $\mathrm{AD}$, reduces to the question of the computation of the function $\beta$.

\section{REFERENCES}

1. H. Becker, A property equivalent to the existence of scales, Trans. Amer. Math. Soc. (to appear).

2. T. Jech, Set theory, Academic Press, New York, 1978.

3. A. S. Kechris, $A D$ and projective ordinals, Cabal Seminar '76-'77 (A. S. Kechris and Y. N. Moschovakis, eds.), Lecture Notes in Math., vol. 689, Springer-Verlag, Berlin and New York, 1978, pp. 91-132.

4. (1984), 255-?67.

5. A coding theorem for measures, mimeographed notes, June 1981.

6. Souslin cardinals, $\kappa$-Souslin sets and the scale property in the hyperprojective hierarchy, Cabal Seminar '77-79 (A. S. Kechris. D. A. Martin and Y. N. Moschovakis, cds.), Lecture Notes in Math., vol. 839, Springer-Verlag, Berlin and New York, 1981, pp. 127-146.

7. A. S. Kechris, E. M. Kleinberg. Y. N. Moschovakis and W. H. Woodin, The Axiom of Determinacv, strong partition properties and nonsingular measures, Cabal Seminar '77-'79 (A. S. Kechris, D. A. Martin and Y. N. Moschovakis, eds.), Lectures Notes in Math., vol. 839, Springer-Verlag, Berlin and New York, 1981, pp. 75-100.

8. A. S. Kechris and W. H. Woodin, The equinalence of partition properties and determinacy, Proc. Nat. Acad. Sci. U.S.A. 80 (1983), 1783-1786.

9. __ The structure of $\Theta$ in inner models of $A D$ (to appear).

10. ____. Generic codes for uncountable ordinals, partition properties and elementary embeddings, mimeographed notes, December 1980.

11. E. M. Kleinberg, Infinitary combinatorics and the Axiom of Determinateness, Lecture Notes in Math., vol. 612, Springer-Verlag, Berlin and New York, 1977.

12. K. Kunen, Some more singular cardinals, mimeographed notes, September 1971.

13. Measurability of $\delta_{n}^{1}$, mimeographed notes, April 1971.

14. __ On $\delta_{5}^{1}$, mimeographed notes, August 1971.

15. __. A remark on Moschovakis' Uniformization Theorem, mimeographed notes, March 1971. 
16. D. A. Martin, The largest countable this, that and the other, Cabal Seminar '79-' 81 (A. S. Kechris, D. A. Martin and Y. N. Moschovakis, eds.) Lecture Notes in Math., vol. 1019, Springer-Verlag, Berlin and New York, 1983, pp. 97-106.

17. , Determinateness implies many cardinals are measurable, mimeographed notes, May 1971.

18. Projective sets and cardinal numbers: Some questions related to the continuum problem, J. Symbolic Logic (to appear).

19. Y. N. Moschovakis, Descriptive set theory, North-Holland, Amsterdam, 1980.

20. J. R. Steel, Scales in $L(\mathbf{R})$, Cabal Seminar '79-'81 (A. S. Kechris, D. A. Martin and Y. N. Moschovakis, eds.) Lecture Notes in Math., vol. 1019, Springer-Verlag, Berlin and New York, 1983, pp. 107-156.

21. Closure properties of pointclasses, Cabal Seminar '77-'79 (A. S. Kechris, D. A. Martin and V. N. Moschovakis, eds.), Lecture Notes in Math., vol. 839, Springer-Verlag, Berlin and New York, 1981, pp. 147-164.

Department of Mathematics, California Institute of Technology, Pasadena, California 91125 